\title{
Buchbesprechungen - Book Reviews - Livres Nouveaux
}

nahmen für die Behandlung der Arteriosklerose der Extremitäten werden fast lückenlos angeführt, einzig auf die intraarterielle Histamintherapie wird nicht eingegangen. Besonderes Gewicht wird $u$. a. auf die von Loeper eingeführte intraarterielle Sauerstoffbehandlung und die Operationsindikationen und $\bullet$ methoden gelegt. Wenn auch die zitierte Literatur zum größten Teil auf französische Autoren beschränkt ist, so stellt doch die concis abgefaßte Monographie ein sehr wertvolles Hilfsmittel für das praktische Vorgehen beim Arteriosklerotiker dar. B. Steinmann, Bern. B. Steinmann und P. Imhof: Die Behandlung der Hemiplegie. Huber, Bern und Stuttgart 1955. 122 Seiten, 50 Abb. Fr. 15.20.

Bei der Häufigkeit zerebraler Gefäí3erkrankungen, die nach Angaben von Brain in England 14\% aller Todesfälle ausmachen, ist es sehr zu begrüßen, daß die Autoren versucht haben, mit ihrer Arbeit zur Klärung der zahlreichen, in der Praxis oft etwas vernachlässigten diagnostischen und therapeutischen Probleme beizutragen. Es ist ihnen dies mit der kleinen, sehr klar und kritisch geschriebenen Monographie vollauf gelungen.

Nach einer einleitenden Übersicht der Physiologie, Pharmakologie und Pathophysiologie des Hirnkreislaufes wird die oft recht schwierige Differen-tialdiagnose der Hauptformen zerebraler vaskulärer Störungen (lokale Throm-bose, Blutung, Embolie) ausführlich besprochen. Wichtig sind die Hinweise auf die relative Seltenheit der Massenblutungen gegenüber den Erweichungen (15\% : 85\%) sowie auf den Wert der frühzeitig vorgenommenen Lumbalpunk-tion, wobei normaler Druck und normale Beschaífenheit des Liqviors für einen Gefäßverschluß sprechen. Hauptthema der Schrift bildet die Behandlung der zerebralen Hemiplegie. Die verschiedenen Möglichkeiten der medikamentösen Therapie werden ausführlich und kritisch besprochen. Auf Grund ihrer eigenen Erfahrungen und der Literaturangaben kommen die Autoren bei den Gefäßverschlüssen zur Empfehlung einer intensiven Frühbehandlung mit Euphyllin oder Niconacid, die während mehreren Wochen fortgesetzt werden muß. Demgegenüber ist die Indikation der Therapie mit Antikoagulantien klinisch oft schwierig zu stellen, ihre Wirksamkeit unsicher und nicht ohne Risiko.

Die Wiederherstellungsbehandlung, der wichtigste Teil der Therapie, hat möglichst frühzeitig mit sorgfältiger Lagerung und passiven Bewegungen der gelähmten Glieder einzusetzen. Bei den folgenden aktiven Bewegungsübungen ist großes Gewicht auf die psychische Führung des Patienten zu legen, der möglichst bald zur Ausführung von praktisch wichtigen Zweckbewegungen an-gehalten werden muß. Zahlreiche Skizzen erläutern die Ausführungen über die Bewegungstherapie. Damit wird die Schrift nicht nur für den Arzt, sondern auch für den Physiotherapeuten zu einer wertvollen Hilfe in dem praktisch so wichtigen Gebiet zerebraler Durchblutungsstörungen.

F. Kesselring, St. Gallen. 